MATHEMATICS OF COMPUTATION

Volume 80, Number 274, April 2011, Pages 1041-1052

S $0025-5718(2010) 02424-X$

Article electronically published on October 22, 2010

\title{
COMPUTING TOTALLY POSITIVE ALGEBRAIC INTEGERS OF SMALL TRACE
}

\author{
JAMES MCKEE
}

\begin{abstract}
We construct minimal polynomials of totally positive algebraic integers of small absolute trace by consideration of their reductions modulo auxiliary polynomials. Many new examples of such polynomials of minimal absolute trace (for given degree) are found. The computations are pushed to degrees that previously were unattainable, and one consequence is that the new examples form the majority of all those known. As an application, we produce a new bound for the Schur-Siegel-Smyth trace problem.
\end{abstract}

\section{INTRODUCTION}

1.1. The absolute trace of a totally positive algebraic integer. Let $\theta$ be a totally positive algebraic integer of degree $d$ with minimal polynomial

$$
P(x)=x^{d}-a_{d-1} x^{d-1}+a_{d-2} x^{d-2}-\cdots+(-1)^{d} a_{0} .
$$

Here the $a_{i}$ are necessarily all positive integers, given that all the Galois conjugates of $\theta$ are real and positive. The absolute trace of $\theta$ is the quantity $a_{d-1} / d$, namely the arithmetic mean of the roots of $P(x)$. This cannot be too small: the product of all the roots of $P(x)$ is a positive integer, so it is at least 1 , and then the arithmeticgeometric mean inequality shows that $a_{d-1} \geq d$, so that the absolute trace is at least 1 . Moreover, the absolute trace is greater than 1 unless $P(x)=x-1$.

We shall see that for degree at least 5 , one has

$$
a_{d-1} \geq\lceil 1.78839 d\rceil \text {. }
$$

This bound is known to be sharp for $d=5,6,7,8,9,10,12,13,14$ (see $\$ 3.2$ for the last of these). It is probably not sharp for $d=11$. Minimal traces for degrees below 5 were provided by Smyth [13].

1.2. The Schur-Siegel-Smyth trace problem. Siegel 12 observed that 2 is a limit point of the set of absolute traces, on considering the totally positive algebraic integers $4 \cos ^{2}(2 \pi / m)$ for $m \in \mathbb{N}$. What is the smallest limit point? This is the Schur-Siegel-Smyth trace problem. Schur [1] showed that the smallest limit point is at least $\sqrt{e} \approx 1.6487$, Siegel [12] improved this bound to 1.7336, and Smyth [13, 14] improved it further to 1.7719. More recent improvements are mentioned in the next subsection, and indeed in recent years no record has lasted for very long. Any examples of totally positive algebraic integers with absolute trace below 2, other than Siegel's 'cosine' examples are therefore of some interest, and the majority of

Received by the editor December 23, 2009 and, in revised form, February 22, 2010.

2010 Mathematics Subject Classification. Primary 11R04, 11Y40.

(C)2010 American Mathematical Society Reverts to public domain 28 years from publication 
the known examples with degree below 15 have been found using the new method described in the current paper.

1.3. Smyth's attack on the trace problem. Since his key paper in 1984 [13, all improvements to the bound in the trace problem have come from Smyth's method, which we now recall briefly. First one finds a collection of 'good' polynomials $Q_{i}(x)$ (more details as to what 'good' might mean will be given below), then one uses semi-infinite linear programming or some other optimisation technique to establish an inequality of the shape

$$
x-\sum_{i \in I} \alpha_{i} \log \left|Q_{i}(x)\right| \geq \rho, \quad x>0,
$$

for some subset of the $Q_{i}$, indexed by $I$, and where the $\alpha_{i}$ are positive real constants, chosen to maximise $\rho$.

Averaging (3) over all the roots of $P(x)$, one obtains

$$
\frac{a_{d-1}}{d} \geq \rho+\sum_{i} \frac{\alpha_{i}}{d} \log \left|\operatorname{Res}\left(P, Q_{i}\right)\right| .
$$

Here $\operatorname{Res}(A, B)$ denotes the resultant of $A$ and $B$. If $P$ as in (1) has roots $\theta_{1}$, $\ldots, \theta_{d}$, then $\operatorname{Res}(P, B)=B\left(\theta_{1}\right) \cdots B\left(\theta_{d}\right)$. This uses that $P$ is monic, but $B$ need not be. If $P$ has no root in common with any of the $Q_{i}$ (assumed to have integer coefficients), then each resultant in (4) has absolute value at least 1 , and hence the absolute trace is at least $\rho$.

Using a variety of selections of polynomials $Q_{i}$, several improvements on the value of $\rho$ have been made. In each case, the improvement resulted from using new 'good' polynomials. Smyth [13, 14] obtained the bound 1.7719 drawing on polynomials of degree $d$ up to 7 and trace at most $d+6$. Flammang, Grandcolas and Rhin 5], used cosine polynomials and transforms of small Salem polynomials to improve this to 1.7735. The author and Smyth [7] found all minimal trace polynomials of degree 10 (the smallest degree $d$ for which the trace is as small as $2 d-2$ ), and used these to improve the bound to 1.7783. Aguirre, Bilbao and Peral [1] performed extensive computations to find good auxiliary polynomials $Q_{i}$ and improved the bound to 1.7800 . In particular, they used a minimal-trace polynomial of degree 13 (the first such appeared in transformed form in 7]). A further improvement by Aguirre and Peral to 1.7841 2 exploited four new polynomials of degree 12 and trace 22 (minimal). Flammang 4 made use of polynomials with not all roots real to improve the bound to 1.78702 ; these new polynomials were found using Wu's algorithm [16].

Smyth 15 showed that his method could not push the value of $\rho$ in (3) up beyond $2-10^{-41}$. In a letter to Smyth (mentioned in [15, and published as an appendix to [2]), Serre showed that Smyth's method could not produce a bound better than $1.898302 \ldots$. . In practice, getting close to this number by Smyth's method is infeasible, since (as we shall see) there are so many examples of polynomials that have absolute trace below Serre's number. Perhaps the smallest limit point of the set of absolute traces is strictly smaller than 2, and perhaps even smaller than Serre's number.

1.4. Plan for this paper. Most of the rest of this paper is devoted to a description of a new method to produce totally positive algebraic integers of small absolute trace. Some of the new polynomials found are then used to improve the bound for 
the trace problem. The paper concludes with a discussion of potential applications and challenges for future research.

All computations were performed using PARI/GP [9].

\section{THE METHOD}

2.1. Bounds on resultants. As observed by Flammang and Rhin [6], the inequality (4) bounds $\left|\operatorname{Res}\left(P, Q_{i}\right)\right|$. Their technique for finding polynomials with constrained roots is to bound the sums of the powers of the roots, using auxiliary functions much as in (3). Bounds for the coefficients are deduced using Newton's identities. In [6], in cases when the computation would otherwise have been too lengthy, they used resultant bounds to reduce the possibilities for the lower-order coefficients.

The method in this paper pushes this idea to the extreme, using mainly resultant information, and relatively little (if any) looping over possible coefficients. The heuristic used for the new method is to choose a small number of low-degree monic $Q_{i}$ that are small on $[0,4]$, and then find $P$ that has small resultant with all the $Q_{i}$. This amounts to finding a small resultant of $P$ with the product of the $Q_{i}$, but there is considerable computational benefit to working with the $Q_{i}$ separately.

For any polynomial $P$ as in (1), and any monic auxiliary polynomial $Q_{i} \in \mathbb{Z}[x]$, define $P_{i}$ to be the reduction of $P$ modulo $Q_{i}$. Note that $\operatorname{Res}\left(P, Q_{i}\right)=\operatorname{Res}\left(P_{i}, Q_{i}\right)$. This resultant should be small, and the coefficients of $P_{i}$ not too large.

This suggests a very naive search method: for a collection of 'good' $Q_{i}(x)$, guess the values of each $P_{i}$, and glue together via the Chinese Remainder Theorem. One could also easily combine this with Robinson's algorithm ([10, and see also [7]). A more intelligent search, making use of a little algebraic number theory, is detailed in $\$ 2.2$ A heuristic speed-up, based on an empirical observation of which residues actually appear, is then described in 92.3 . A theoretical explanation of the observed phenomenon is also given in that section.

Note that not all lists of candidates for the $P_{i}$ lift to give $P$ with integer coefficients, and this can cut out many cases at an early stage. This was noted in [6] in the context of linear $Q_{i}$. Indeed, one gets a lattice of legitimate reduced polynomials, but the additive structure of this lattice does not fit well with the multiplicative structure of those polynomials that have small resultants with $Q_{i}$.

2.2. Resultants and norms. We shall assume that all of our $Q_{i}$ are irreducible and monic. Let $\theta_{i}$ be a chosen root of $Q_{i}$. We use the standard fact (e.g., 3. Proposition 4.3.4]) that the resultant of $P_{i}$ and $Q_{i}$ is (plus or minus) the norm of $P_{i}\left(\theta_{i}\right)$. For resultant \pm 1 , we are looking for units in $\mathbb{Q}\left(\theta_{i}\right)$. For small resultants, we want elements of small norm, $N_{\mathbb{Q}\left(\theta_{i}\right) / \mathbb{Q}}\left(P_{i}\left(\theta_{i}\right)\right)$. We summarise these thoughts in the following proposition.

Proposition 1. Let $\left\{Q_{i}\right\}_{i \in I}$ be a finite collection of monic irreducible polynomials in $\mathbb{Z}[x]$, and let $\left\{\alpha_{i}\right\}_{i \in I}$ be a corresponding set of positive real numbers, and let $\rho \in \mathbb{R}$ be such that (3) holds. For each $i \in I$, let $\theta_{i}$ be a root of $Q_{i}$. Then for any totally positive monic polynomial $P(x) \in \mathbb{Z}[x]$ of degree $d$ and absolute trace $t / d$, and with $P$ not divisible by any of the $Q_{i}$, there holds for each $i \in I$,

$$
\left|N_{\mathbb{Q}\left(\theta_{i}\right) / \mathbb{Q}}\left(P_{i}\left(\theta_{i}\right)\right)\right| \leq \exp \left((t-d \rho) / \alpha_{i}\right),
$$

where $P_{i}$ is the reduction of $P$ modulo $Q_{i}$. 
This suggests a more intelligent search method: for each $Q_{i}$, precompute a list of units in $\mathbb{Q}\left(\theta_{i}\right)$ with coefficients up to some bound, and a set of inequivalent elements of small norm. Use these to search through possible values of $P_{i}$.

Let us illustrate the idea by considering the problem of finding all degree-10 totally positive algebraic integers of trace 18 (a computation that was performed in [7] in 150 hours on a single processor (with the same code running in 35 hours on a fast modern laptop), but that now can be verified much more quickly). We choose $Q_{i}$ whose degrees sum to $9=d-1$, or close to it: any shortfall will be made up by looping over certain coefficients. Here a good choice is to use $Q_{1}, Q_{2}, Q_{3}, Q_{4}$, $Q_{7}$ from Table 1, with a degree sum of 8, and loop over the possible values of $a_{8}$ in (11). Using Proposition 1 with the value of $\rho=1.78839$ following from the selection of polynomials and coefficients in Table 1, we find that the absolute value of the resultant of our putative degree-10 polynomial $P$ with each of these five auxiliary polynomials is at most $1,1,4,1,5$ (here assuming that $P$ is not one of $Q_{25}, Q_{26}$, $Q_{27}$, so that we can apply Proposition 1). Thus the constant term of $P$ must be 1 , the remainder on dividing $P$ by $x-1$ must be \pm 1 , and the remainder on dividing $P$ by $x-2$ lies between -4 and 4 (and is not 0 ). The resultant with $x^{2}-3 x+1$ must be \pm 1 , so that the residue of $P$ modulo $x^{2}-3 x+1$ is a unit in the relevant quadratic field, so it is plus or minus an integer power of $x-1$ (modulo $x^{2}-3 x+1$ ). Luckily the cubic field defined by $Q_{7}$ has no nonunits for which the modulus of the norm is as small as 5, so that we are just looking at plus or minus products of integer powers of generators of the unit group: $x^{2}-3 x+1$ and $x-1$.

To continue, we need to obtain bounds for the leading coefficient of the reduction of $P$ modulo each of the nonlinear auxiliary polynomials, $Q_{4}$ and $Q_{7}$. One can get fairly weak bounds quite simply. The coefficients $a_{0}, \ldots, a_{8}$ in (11) are bounded above by the corresponding coefficients of $(x-18 / 10)^{10}$. Then one can reduce each relevant power of $x$ modulo each relevant $Q_{i}$ and take appropriately signed linear combinations of these reductions to bound the leading coefficient of $P_{i}$ from above and below. Using the leading coefficient of $P_{i}$, one can bound the powers of the relevant units. The search was completed in 3.5 hours, taking only $10 \%$ of the previous time on the same machine. Now see the final paragraph of $\$ 2.3$ for an even more dramatic reduction in the time, but without proof of completeness.

2.3. Good residues and bad. One rapidly observes that some residues modulo some $Q_{i}$ are much more prevalent than others of the same norm. Being able to predict in advance which residues are more likely to occur greatly speeds the search: one can find the majority of solutions in a tiny fraction of the time.

Define

$$
m_{i}=Q_{i}(x) /\left(x-\theta_{i, \max }\right),
$$

where $\theta_{i, \max }$ is the largest root of $Q_{i}(x)$ (all the $Q_{i}$ used will be totally real, although in the spirit of Flammang's work [4 one might wish to relax this constraint). If $P_{i}$ is close to a real multiple of $m_{i}$, then its resultant with $Q_{i}$ will be small. It was observed that without exception the values of $P_{i}$ for some 106 degree-12 $P$ that had been computed using the method of $\$ 2.2$ were close to a multiple of $m_{i}$. This provided a much faster way of performing the search: loop through multiples of $m_{i}$, round coefficients to the nearest integer, and allow small perturbations of the coefficients. 
One can explain this phenomenon for the specific $Q_{i}$ used as follows. We treat the quadratic case $Q_{i}=Q_{4}=x^{2}-3 x+1$ in detail (other cases are similar). Let $\alpha, \beta$ be the roots of $Q_{4}$, with $\alpha<\beta$. Note that $\beta$ is actually a Pisot number, since $|\alpha|<1<\beta$. Write (for $j \geq 0) x^{j} \equiv r_{j} x+s_{j}\left(\bmod Q_{4}\right)$. Then one obtains recurrence relations for the $r_{j}$, with solution

$$
r_{j}=\frac{\alpha^{j}-\beta^{j}}{\alpha-\beta}, \quad s_{j}=\frac{\beta^{j-1}-\alpha^{j-1}}{\alpha-\beta} .
$$

In particular, we see that $r_{j} x+s_{j}=0$ has root $\left(\alpha^{j-1}-\beta^{j-1}\right) /\left(\alpha^{j}-\beta^{j}\right)$. Since $\alpha^{j} \rightarrow 0$ as $j \rightarrow \infty$, this root approaches $1 / \beta=\alpha$. Moreover, this root approaches $\alpha$ from below. Since one can easily show that $a_{1}>a_{0}$, we conclude that $P_{4}$, the reduction of $P$ modulo $Q_{4}$, is a sum of linear polynomials with roots between 0 and $\alpha$ : given that this sum must have its root close to either $\alpha$ or $\beta$, it is no surprise that $\alpha$ is favoured.

We can illustrate this heuristic variant by returning to the degree 10 , trace 18 problem. Choosing $Q_{1}, Q_{2}, Q_{3}, Q_{4}, Q_{7}$ with resultant bounds all 1, leading coefficient bounds $1,1,8,1,16$ (respectively), and no perturbation of the coefficients, all three polynomials were found within 2 seconds(!), looping over $a_{8}$, but of course without proof that the list is complete.

\section{Results}

3.1. Degree 9 revisited. It is known [2] that there are 686 irreducible polynomials of degree 9 with all roots real and positive, and trace 17 (the smallest possible for degree 9). Can our method find them all?

First we select auxiliary polynomials with degree sum 8 (or close to it), say $Q_{1}, Q_{2}, Q_{3}, Q_{4}, Q_{7}$. Letting $d_{i}$ be the degree of $Q_{i}$, the following strategy was employed. Initial bounds for the resultant of $P_{i}$ with $Q_{i}$ were set to be $2^{d_{i}}$, with the same initial bounds for the leading coefficients; for nonlinear $Q_{i}$ initial permitted perturbations of each coefficient were set to 1 . For each parameter in turn, the parameter was repeatedly doubled and the method of $\$ 2.3$ applied until no new polynomials were found; then the parameter being varied was reduced to the largest observed value. The parameters were cycled through, being treated in this way, until no new polynomials were produced. The final parameters, from which all 686 polynomials were found, are shown in the following table.

\begin{tabular}{|l|l|l|l|l|l|}
\hline polynomial & $x$ & $x-1$ & $x-2$ & $x^{2}-3 x+1$ & $x^{3}-5 x^{2}+6 x-1$ \\
\hline \hline resultant bound & 3 & 3 & 23 & 11 & 83 \\
\hline leading coefficient bound & 3 & 3 & 23 & 55 & 133 \\
\hline $\begin{array}{l}\text { permitted perturbation } \\
\text { of coefficients }\end{array}$ & 0 & 0 & 0 & 1 & 8 \\
\hline
\end{tabular}

The rationale for this approach was that any missing polynomials would be extreme outliers with respect to one or more of the parameters. Encouraged by this success for degree 9 , the same strategy was employed to produce the 209 degree-12 trace-22 examples mentioned in 4.1 .

3.2. New minimal-trace examples. For degrees 12,13 , 14, the minimal trace is, respectively, 22, 24, 26. Prior to this work, only a handful of examples were known ([7, [1], 2]), with none for degree 14. For degree 12, see $\$ 4.1$ below, where the list of known examples is extended to 209 in number. 
For degree 13 , trace 24 , take the auxiliary polynomials $Q_{1}, Q_{2}, Q_{3}, Q_{4}, Q_{7}, Q_{11}$. Using the method of 2.3 , some 1148 polynomials were found within a few days. Similarly, for degree 14, trace 26, some 3872 polynomials were found. Since none of these has appeared in print before, we celebrate by presenting one:

$$
\begin{aligned}
& x^{14}-26 x^{13}+298 x^{12}-1984 x^{11}+8513 x^{10}-24701 x^{9}+49494 x^{8}-68757 x^{7} \\
& \quad+65607 x^{6}-42064 x^{5}+17471 x^{4}-4437 x^{3}+627 x^{2}-42 x+1 .
\end{aligned}
$$

The online version of this paper contains a link to lists of all these minimal-trace examples.

3.3. New examples with absolute trace below 2. We can apply the construction to other nonminimal (or possibly nonminimal) cases: degree 10 and trace 19, degree 11 and trace 21 (perhaps minimal), degree 12 and trace 23, degree 13 and trace 25 , degree 14 and trace 27 . Thousands more examples are easily found.

For degree 10 and trace 19, the auxiliary polynomials used were $Q_{1}, Q_{2}, Q_{3}, Q_{4}$, $Q_{7}$, with resultant bounds $2,3,4,16,32$; for $Q_{4}$ and $Q_{7}$, the bounds for the leading coefficients of the $P_{i}$ were, respectively, 64 and 128, and the permitted perturbations of the nonleading coefficients of the rounded integer multiples of $m_{4}$ and $m_{7}$ were, respectively, 1 and 2 . The sum of the degrees of the auxiliary polynomials is only 8; this shortfall was made up by looping over $a_{8}$ in the range 140-155.

For degree 11 and trace 21, the auxiliary polynomials chosen were $Q_{1}, Q_{2}, Q_{3}$, $Q_{4}, Q_{5}, Q_{7}$, with resultant bounds, respectively, 2, 3, 4, 16, 32, 32. For $Q_{4}, Q_{5}, Q_{7}$, the leading coefficients of the $P_{i}$ were bounded by $64,128,128$, and the permitted perturbations of the coefficients were $1,1,2$.

The following table shows the number of polynomials found in each case, including the minimal-trace examples of 33.2 . It should be stressed that these searches were not exhaustive, and the numbers given are lower bounds for the total number of examples.

\begin{tabular}{|c|c|c|}
\hline degree & trace & number of examples found \\
\hline 10 & 19 & 2503 \\
11 & 21 & 8496 \\
12 & 22 & 209 \\
13 & 24 & 1148 \\
14 & 26 & 3872 \\
\hline
\end{tabular}

\section{A NEW LOWER BOUND FOR THE TRACE PROBlem}

4.1. New auxiliary polynomials. Using the method of $\$ 2.3$ and the strategy of 3.1 some 209 polynomials of degree 12 and trace 22 were found; previously 4 of these had appeared in the literature [2]. The auxiliary polynomials used were $Q_{1}$, $Q_{2}, Q_{4}, Q_{7}, Q_{11}$ from Table 1 . The bounds on the resultants were 1, 1, 5, 13, 139 . The bounds on the leading coefficients of the residues were $1,1,34,136,1207$. The bounds on the permitted perturbations of the coefficients were $0,0,0,4,5$.

4.2. The linear programming. Adding our 209 degree 12 trace 22 totally positive polynomials to Flammang's list of 35 polynomials, linear programming was used to obtain the lower bound advertised in (2). The $Q_{i}$ and $\alpha_{i}$ are tabulated in Table 1. A word about the confidence in the numerical accuracy of this bound is in order. One can use calculus to identify the position of the local minima of the function on the left of (3) to any desired accuracy; but how confident can we be 
in the function values at these local minima? This question is simplified by the following lemma.

Lemma 2. With the $Q_{i}$ and $\alpha_{i}$ given in Table 1 , define the function $F(x)$ for $x>0$ by

$$
F(x)=x-\sum_{i=1}^{70} \alpha_{i} \log \left|Q_{i}(x)\right| .
$$

Then $F^{\prime \prime}(x)>0$ for all positive $x$ for which it is defined.

Proof. Define $g_{i}(x)=\log \left|Q_{i}(x)\right|$. We have $F^{\prime \prime}(x)=-\sum \alpha_{i} g_{i}^{\prime \prime}(x)$. Now if $Q_{i}$ is totally real, then since

$$
g_{i}^{\prime}(x)=\frac{Q_{i}^{\prime}(x)}{Q_{i}(x)}=\sum \frac{1}{x-\theta_{i, k}},
$$

where the $\theta_{i, k}$ are the roots of $Q_{i}$, the contribution to $F^{\prime \prime}$ from such $Q_{i}$ is strictly positive.

But some of Flammang's polynomials are not totally real, and indeed the corresponding $g_{i}^{\prime \prime}(x)$ do change sign. To cope with these, the roots of all these troublesome $g_{i}^{\prime \prime}$ were computed, and the real line was split into intervals, in each of which at most three of the $g_{i}^{\prime \prime}$ were positive (hence contributing negatively to $F^{\prime \prime}$ ). In each such interval, a small set of totally real $Q_{i}$ was found such that their contribution to $F^{\prime \prime}$ dominated those of the awkward $g_{i}^{\prime \prime}$. In this way one could establish that $F^{\prime \prime}$ had no real roots without having to deal with polynomials of impossibly large degree. For example, in the interval [0,0.15], the troublesome Flammang polynomials are $Q_{17}, Q_{29}$ and $Q_{34}$; in this interval, one can check (using Sturm's theorem) that $-\alpha_{1} g_{1}-\alpha_{17} g_{17}-\alpha_{29} g_{29}-\alpha_{34} g_{34}$ has positive second derivative throughout the interval, and hence so does $F$.

Content that $F^{\prime \prime}(x)>0$ for $x>0$, we now split the positive real axis into 631 subintervals, with endpoints given by the roots of all the $Q_{i}$ (and also $\infty$ ). In each subinterval $I$, a bisection technique was used to find three points $x_{1}=x_{2}-h<$ $x_{2}<x_{3}=x_{2}+h$ with $F\left(x_{2}\right)<\max \left\{F\left(x_{1}\right), F\left(x_{3}\right)\right\}$, and with all the $F\left(x_{i}\right)$ very close. Then in $I$ a lower bound for $F$ is given by $2 F\left(x_{2}\right)-\max \left\{F\left(x_{1}\right), F\left(x_{3}\right)\right\}$. For the polynomials and coefficients given in Table 1 , the smallest of these lower bounds over all the subintervals $I$ was 1.78839 , giving (2).

\section{Applications And Challenges}

5.1. Degrees 11 and 15. Extensive searches have failed to find any examples with degree 11 and trace 20 . The method of 2.2 , with more work on bounding resultants and coefficients, could surely polish off this case. But the possibility of an empty output makes the challenge relatively unexciting. Similar comments hold for degree 15 and trace 27 , although a complete search using the method of \$2.2 is considerably more daunting. A more straightforward challenge is to update and extend the table in 93.3

5.2. Variants of the method. For each degree $d$, one might consider several subsets of the $Q_{i}$ for which the sum of the degrees was close to $d-1$. One could then run the method of 2.3 on each subset, and pool the results. Keeping the bounds moderately small would greatly speed the search. The hope would be that nearly all polynomials would fall into one of the subsets considered, so that 
a majority of all possible examples would be found in a fraction of the time. For degree/trace combinations where a more systematic search would be impossible, this might be an attractive variant.

Rather than precomputing lists of potentially good residues that might never be used, an alternative strategy of growing lists of popular residues might be profitable for large degrees. Start with lists $L_{i}$ such that small-trace polynomials are known with $P_{i} \in L_{i}$. Search for more polynomials using only these 'good' residues. Any new polynomials found via auxiliary polynomials $Q_{i}(i \in I)$ can be used to grow the lists $L_{j}$ for $j \notin I$.

5.3. Improving the rigorous variant. If the method is to be used for an exhaustive search, then the resultant bounds should be the best-known derived from expressions of the form (4), and coefficient bounds need to be rigorously derived. One improvement would be to do the right optimisation for bounding each resultant, rather than doing a single global optimisation for $\rho$; one might find a smaller value of $\rho$ which gives a better bound for the resultant of $P$ with $Q_{i}$ because the value of $\alpha_{i}$ is larger.

5.4. Trace $2 d-3$. What is the smallest $d$ for which there is a totally positive algebraic integer that has degree $d$ and trace $2 d-3$ ? Certainly we have $d \geq 15$. The construction in 8 . for Salem numbers of arbitrary trace produces a Salem number $\tau$ of degree 76 and trace -3 . The totally positive algebraic integer $\tau+1 / \tau+2$ has degree 38 and trace $2 d-3=73$. An extension of the interlacing technique in [8] produces an example with degree only 27 :

$$
\begin{aligned}
& x^{27}-51 x^{26}+1223 x^{25}-18339 x^{24}+192898 x^{23}-1513373 x^{22}+9192976 x^{21} \\
& \quad-44303101 x^{20}+172186638 x^{19}-545699142 x^{18}+1420423645 x^{17}-3049383852 x^{16} \\
& \\
& +5408448828 x^{15}-7920958921 x^{14}+9554306801 x^{13}-9447108297 x^{12}+7604508649 x^{11} \\
& \\
& \quad+4936587363 x^{10}+2552735579 x^{9}-1034886788 x^{8}+322250908 x^{7}-75059956 x^{6} \\
& \quad+12632359 x^{5}-1466911 x^{4}+110409 x^{3}-4934 x^{2}+114 x-1 .
\end{aligned}
$$

Applying the method of 2.3 optimistically one might discover an example of even smaller degree.

5.5. Discriminants of totally real polynomials. Totally real polynomials of small absolute trace have relatively small discriminants. The techniques of this paper could be applied to the search for small discriminants of totally real polynomials.

\section{Appendix: the polynomials and Coefficients for the bound (2)}

Here we list the 70 polynomials $Q_{1}, \ldots, Q_{70}$ and their corresponding coefficients that were selected from 244 possible polynomials by the linear programming routine. They are ordered by degree, and then by the values of their coefficients. Of the 70 polynomials, 32 appeared in Flammang's list [4, and the remaining 38 are from the new list of 209 degree-12 trace-22 polynomials (three of the four used by Aguirre and Peral [2] were selected: numbers 32, 33, 46). All computations were performed using a precision of 28 significant digits; the coefficients are displayed only to 10 decimal places. 
Table 1: Auxiliary polynomials and their coefficients for the bound (2)

\begin{tabular}{|c|c|c|}
\hline$i$ & $Q_{i}$ & $\alpha_{i}$ \\
\hline 1 & $x$ & 0.5458822075 \\
\hline 2 & $x-1$ & 0.4768270490 \\
\hline 3 & $x-2$ & 0.0732017208 \\
\hline 4 & $x^{2}-3 x+1$ & 0.1796348117 \\
\hline 5 & $x^{2}-4 x+2$ & 0.0095848069 \\
\hline 6 & $x^{2}-4 x+1$ & 0.0060806088 \\
\hline 7 & $x^{3}-5 x^{2}+6 x-1$ & 0.0684812443 \\
\hline 8 & $x^{3}-6 x^{2}+9 x-1$ & 0.0062431622 \\
\hline 9 & $x^{3}-6 x^{2}+9 x-3$ & 0.0034242075 \\
\hline 10 & $x^{3}-6 x^{2}+8 x-1$ & 0.0004435369 \\
\hline 11 & $x^{4}-7 x^{3}+13 x^{2}-7 x+1$ & 0.0302909976 \\
\hline 12 & $x^{4}-7 x^{3}+14 x^{2}-8 x+1$ & 0.0239553796 \\
\hline 13 & $x^{5}-9 x^{4}+27 x^{3}-31 x^{2}+12 x-1$ & 0.0075885021 \\
\hline 14 & $x^{5}-9 x^{4}+28 x^{3}-35 x^{2}+15 x-1$ & 0.0048599436 \\
\hline 15 & $x^{5}-9 x^{4}+26 x^{3}-29 x^{2}+11 x-1$ & 0.0042532436 \\
\hline 16 & $x^{5}-9 x^{4}+27 x^{3}-32 x^{2}+13 x-1$ & 0.0022305210 \\
\hline 17 & $x^{6}-10 x^{5}+35 x^{4}-52 x^{3}+33 x^{2}-9 x+1$ & 0.0015266587 \\
\hline 18 & $x^{6}-11 x^{5}+44 x^{4}-78 x^{3}+59 x^{2}-15 x+1$ & 0.0002437391 \\
\hline 19 & $x^{7}-13 x^{6}+63 x^{5}-143 x^{4}+157 x^{3}-78 x^{2}+16 x-1$ & 0.0010027729 \\
\hline 20 & $x^{8}-15 x^{7}+90 x^{6}-277 x^{5}+467 x^{4}-428 x^{3}+200 x^{2}-42 x+3$ & 0.0015333021 \\
\hline 21 & $x^{8}-14 x^{7}+78 x^{6}-221 x^{5}+339 x^{4}-277 x^{3}+111 x^{2}-19 x+1$ & 0.0014339129 \\
\hline 22 & $x^{8}-15 x^{7}+89 x^{6}-269 x^{5}+445 x^{4}-402 x^{3}+187 x^{2}-40 x+3$ & 0.0011925457 \\
\hline 23 & $x^{8}-14 x^{7}+78 x^{6}-222 x^{5}+345 x^{4}-289 x^{3}+120 x^{2}-21 x+1$ & 0.0006074869 \\
\hline 24 & $\begin{array}{l}x^{9}-16 x^{8}+103 x^{7}-345 x^{6}+651 x^{5}-703 x^{4}+424 x^{3}-135 x^{2} \\
\quad+20 x-1\end{array}$ & 0.0020620779 \\
\hline 25 & $\begin{array}{l}x^{10}-18 x^{9}+135 x^{8}-549 x^{7}+1320 x^{6}-1920 x^{5}+1662 x^{4}-813 x^{3} \\
\quad+206 x^{2}-24 x+1\end{array}$ & 0.0054614574 \\
\hline 26 & $\begin{array}{l}x^{10}-18 x^{9}+134 x^{8}-538 x^{7}+1273 x^{6}-1822 x^{5}+1560 x^{4}-766 x^{3} \\
\quad+200 x^{2}-24 x+1\end{array}$ & 0.0035987602 \\
\hline 27 & $x^{10}-18 x^{9}+134 x^{8}-537 x^{7}+1265 x^{6}-1798 x^{5}+1526 x^{4}-743 x^{3}$ & 00030737869 \\
\hline 28 & $\begin{array}{l}x^{10}-18 x^{9}+135 x^{8}-550 x^{7}+1331 x^{6}-1967 x^{5}+1760 x^{4}-916 x^{3} \\
\quad+256 x^{2}-32 x+1\end{array}$ & 0.0013072598 \\
\hline 29 & $\begin{array}{l}x^{12}-21 x^{11}+190 x^{10}-972 x^{9}+3103 x^{8}-6439 x^{7}+8780 x^{6} \\
\quad-7789 x^{5}+4372 x^{4}-1483 x^{3}+283 x^{2}-27 x+1\end{array}$ & 0.0073877754 \\
\hline 30 & $\begin{array}{l}x^{12}-21 x^{11}+190 x^{10}-972 x^{9}+3102 x^{8}-6430 x^{7}+8750 x^{6} \\
-7742 x^{5}+4336 x^{4}-1470 x^{3}+281 x^{2}-27 x+1\end{array}$ & 0.0037158977 \\
\hline 31 & $\begin{array}{l}x^{12}-22 x^{11}+208 x^{10}-1108 x^{9}+3666 x^{8}-7840 x^{7}+10948 x^{6} \\
\quad-9877 x^{5}+5589 x^{4}-1885 x^{3}+349 x^{2}-31 x+1\end{array}$ & 0.0023864597 \\
\hline 32 & $\begin{array}{l}x^{12}-22 x^{11}+207 x^{10}-1092 x^{9}+3561 x^{8}-7474 x^{7}+10213 x^{6} \\
\quad-9012 x^{5}+5007 x^{4}-1676 x^{3}+314 x^{2}-29 x+1\end{array}$ & 0.0023448210 \\
\hline 33 & $\begin{array}{l}x^{12}-22 x^{11}+204 x^{10}-1050 x^{9}+3322 x^{8}-6752 x^{7}+8944 x^{6} \\
-7677 x^{5}+4177 x^{4}-1388 x^{3}+265 x^{2}-26 x+1\end{array}$ & 0.0016259610 \\
\hline 34 & $\begin{array}{l}x^{12}-21 x^{11}+189 x^{10}-958 x^{9}+3023 x^{8}-6196 x^{7}+8352 x^{6} \\
\quad-7341 x^{5}+4097 x^{4}-1389 x^{3}+267 x^{2}-26 x+1\end{array}$ & 0.0012617180 \\
\hline 35 & $\begin{array}{l}x^{12}-22 x^{11}+207 x^{10}-1093 x^{9}+3575 x^{8}-7549 x^{7}+10410 x^{6} \\
\quad-9284 x^{5}+5204 x^{4}-1748 x^{3}+327 x^{2}-30 x+1\end{array}$ & 0.0012560669 \\
\hline
\end{tabular}


Table 1: (continued) Auxiliary polynomials and coefficients.

\begin{tabular}{|c|c|c|}
\hline$i$ & $2_{i}$ & $\alpha_{i}$ \\
\hline 36 & $\begin{array}{l}x^{12}-22 x^{11}+207 x^{10}-1094 x^{9}+3587 x^{8}-7607 x^{7}+10558 x^{6} \\
\quad-9501 x^{5}+5387 x^{4}-1830 x^{3}+343 x^{2}-31 x+1\end{array}$ & 0.0010625610 \\
\hline 37 & $\begin{array}{l}x^{12}-22 x^{11}+206 x^{10}-1075 x^{9}+3443 x^{8}-7042 x^{7}+9313 x^{6} \\
\quad-7935 x^{5}+4289 x^{4}-1425 x^{3}+274 x^{2}-27 x+1\end{array}$ & 0.0010268366 \\
\hline 38 & $\begin{array}{l}x^{12}-22 x^{11}+208 x^{10}-1108 x^{9}+3667 x^{8}-7851 x^{7}+10995 x^{6} \\
\quad-9977 x^{5}+5702 x^{4}-1952 x^{3}+368 x^{2}-33 x+1\end{array}$ & 0.0008735994 \\
\hline 39 & $\begin{array}{l}x^{12}-22 x^{11}+207 x^{10}-1093 x^{9}+3575 x^{8}-7551 x^{7}+10428 x^{6} \\
-9343 x^{5}+5293 x^{4}-1811 x^{3}+346 x^{2}-32 x+1\end{array}$ & 0.0007246854 \\
\hline 40 & $\begin{array}{l}x^{12}-22 x^{11}+207 x^{10}-1093 x^{9}+3574 x^{8}-7539 x^{7}+10373 x^{6} \\
\quad-9219 x^{5}+5145 x^{4}-1718 x^{3}+318 x^{2}-29 x+1\end{array}$ & 0.0006473624 \\
\hline 41 & $\begin{array}{l}x^{12}-22 x^{11}+207 x^{10}-1093 x^{9}+3573 x^{8}-7529 x^{7}+10336 x^{6} \\
-9154 x^{5}+5088 x^{4}-1695 x^{3}+315 x^{2}-29 x+1\end{array}$ & 0.0005662111 \\
\hline 42 & $\begin{array}{l}x^{12}-22 x^{11}+207 x^{10}-1092 x^{9}+3562 x^{8}-7485 x^{7}+10260 x^{6} \\
-9112 x^{5}+5121 x^{4}-1745 x^{3}+334 x^{2}-31 x+1\end{array}$ & 0.0005486744 \\
\hline 43 & $\begin{array}{l}x^{12}-22 x^{11}+206 x^{10}-1076 x^{9}+3456 x^{8}-7106 x^{7}+9462 x^{6} \\
\quad-8101 x^{5}+4364 x^{4}-1428 x^{3}+268 x^{2}-26 x+1\end{array}$ & 0.0005162692 \\
\hline 44 & $\begin{array}{l}x^{12}-22 x^{11}+206 x^{10}-1077 x^{9}+3470 x^{8}-7182 x^{7}+9666 x^{6} \\
-8391 x^{5}+4582 x^{4}-1511 x^{3}+283 x^{2}-27 x+1\end{array}$ & 0.0003868376 \\
\hline 45 & $\begin{array}{l}x^{12}-22 x^{11}+207 x^{10}-1094 x^{9}+3588 x^{8}-7617 x^{7}+10598 x^{6} \\
-9582 x^{5}+5475 x^{4}-1880 x^{3}+356 x^{2}-32 x+1\end{array}$ & 0.0003838813 \\
\hline 46 & $\begin{array}{l}x^{12}-22 x^{11}+208 x^{10}-1109 x^{9}+3679 x^{8}-7908 x^{7}+11134 x^{6} \\
-10166 x^{5}+5846 x^{4}-2009 x^{3}+377 x^{2}-33 x+1\end{array}$ & 0.0003538106 \\
\hline 47 & $\begin{array}{l}x^{12}-22 x^{11}+208 x^{10}-1108 x^{9}+3665 x^{8}-7829 x^{7}+10902 x^{6} \\
-9784 x^{5}+5492 x^{4}-1834 x^{3}+337 x^{2}-30 x+1\end{array}$ & 0.0002879600 \\
\hline 48 & $\begin{array}{l}x^{12}-22 x^{11}+208 x^{10}-1108 x^{9}+3666 x^{8}-7839 x^{7}+10940 x^{6} \\
-9854 x^{5}+5559 x^{4}-1868 x^{3}+346 x^{2}-31 x+1\end{array}$ & 0.0002180755 \\
\hline 49 & $\begin{array}{l}x^{12}-22 x^{11}+206 x^{10}-1080 x^{9}+3505 x^{8}-7345 x^{7}+10059 x^{6} \\
-8921 x^{5}+4984 x^{4}-1674 x^{3}+314 x^{2}-29 x+1\end{array}$ & 0.0001855736 \\
\hline 50 & $\begin{array}{l}x^{12}-22 x^{11}+208 x^{10}-1109 x^{9}+3679 x^{8}-7907 x^{7}+11124 x^{6} \\
\quad-10130 x^{5}+5787 x^{4}-1964 x^{3}+363 x^{2}-32 x+1\end{array}$ & 0.0001837541 \\
\hline 51 & $\begin{array}{c}x^{12}-22 x^{11}+206 x^{10}-1077 x^{9}+3471 x^{8}-7192 x^{7}+9706 x^{6} \\
-8473 x^{5}+4673 x^{4}-1563 x^{3}+296 x^{2}-28 x+1\end{array}$ & 0.0001826723 \\
\hline 52 & $\begin{array}{l}x^{12}-22 x^{11}+206 x^{10}-1078 x^{9}+3483 x^{8}-7248 x^{7}+9838 x^{6} \\
\quad-8641 x^{5}+4786 x^{4}-1599 x^{3}+300 x^{2}-28 x+1\end{array}$ & 0.0001665401 \\
\hline 53 & $\begin{array}{l}x^{12}-22 x^{11}+205 x^{10}-1065 x^{9}+3412 x^{8}-7035 x^{7}+9455 x^{6} \\
\quad-8221 x^{5}+4513 x^{4}-1501 x^{3}+283 x^{2}-27 x+1\end{array}$ & 0.0001535104 \\
\hline 54 & $\begin{array}{l}x^{12}-22 x^{11}+207 x^{10}-1092 x^{9}+3560 x^{8}-7463 x^{7}+10168 x^{6} \\
\quad-8927 x^{5}+4931 x^{4}-1646 x^{3}+310 x^{2}-29 x+1\end{array}$ & 0.0001390283 \\
\hline 55 & $\begin{array}{l}x^{12}-22 x^{11}+208 x^{10}-1109 x^{9}+3678 x^{8}-7896 x^{7}+11078 x^{6} \\
\quad-10037 x^{5}+5691 x^{4}-1914 x^{3}+351 x^{2}-31 x+1\end{array}$ & 0.0001195768 \\
\hline 56 & $\begin{array}{l}x^{12}-22 x^{11}+207 x^{10}-1092 x^{9}+3560 x^{8}-7464 x^{7}+10176 x^{6} \\
\quad-8948 x^{5}+4952 x^{4}-1654 x^{3}+311 x^{2}-29 x+1\end{array}$ & 0.0001059823 \\
\hline 57 & $\begin{array}{l}x^{12}-22 x^{11}+207 x^{10}-1093 x^{9}+3574 x^{8}-7539 x^{7}+10373 x^{6} \\
-9218 x^{5}+5142 x^{4}-1716 x^{3}+318 x^{2}-29 x+1\end{array}$ & 0.0000879702 \\
\hline 58 & $\begin{array}{l}x^{12}-22 x^{11}+207 x^{10}-1094 x^{9}+3587 x^{8}-7607 x^{7}+10558 x^{6} \\
\quad-9502 x^{5}+5391 x^{4}-1835 x^{3}+345 x^{2}-31 x+1\end{array}$ & 0.0000846965 \\
\hline 59 & $\begin{array}{l}x^{12}-22 x^{11}+207 x^{10}-1096 x^{9}+3611 x^{8}-7723 x^{7}+10850 x^{6} \\
-9914 x^{5}+5717 x^{4}-1972 x^{3}+372 x^{2}-33 x+1\end{array}$ & 0.000 \\
\hline 60 & $\begin{array}{l}x^{12}-22 x^{11}+207 x^{10}-1092 x^{9}+3560 x^{8}-7462 x^{7}+10160 x^{6} \\
\quad-8902 x^{5}+4892 x^{4}-1616 x^{3}+300 x^{2}-28 x+1\end{array}$ & 0.0000747105 \\
\hline
\end{tabular}


Table 1: (continued) Auxiliary polynomials and coefficients.

\begin{tabular}{r|c|c}
$i$ & $Q_{i}$ & $\alpha_{i}$ \\
\hline 61 & $x^{12}-22 x^{11}+206 x^{10}-1077 x^{9}+3468 x^{8}-7164 x^{7}+9608 x^{6}$ & \\
& $-8307 x^{5}+4527 x^{4}-1497 x^{3}+282 x^{2}-27 x+1$ & 0.0000612360 \\
62 & $x^{12}-22 x^{11}+206 x^{10}-1078 x^{9}+3482 x^{8}-7239 x^{7}+9806 x^{6}$ & \\
& $-8584 x^{5}+4733 x^{4}-1575 x^{3}+296 x^{2}-28 x+1$ & 0.0000393296 \\
63 & $x^{12}-22 x^{11}+205 x^{10}-1065 x^{9}+3414 x^{8}-7053 x^{7}+9518 x^{6}$ & \\
& $-8330 x^{5}+4612 x^{4}-1548 x^{3}+294 x^{2}-28 x+1$ & 0.0000371271 \\
64 & $x^{12}-22 x^{11}+209 x^{10}-1124 x^{9}+3772 x^{8}-8218 x^{7}+11740 x^{6}$ & \\
65 & $-10879 x^{5}+6347 x^{4}-2212 x^{3}+421 x^{2}-37 x+1$ & 0.0000253111 \\
66 & $x^{12}-22 x^{11}+208 x^{10}-1108 x^{9}+3665 x^{8}-7829 x^{7}+10903 x^{6}$ & \\
& $-9792 x^{5}+5513 x^{4}-1855 x^{3}+345 x^{2}-31 x+1$ & 0.0000205517 \\
67 & $x^{12}-22 x^{11}+205 x^{10}-1065 x^{9}+3413 x^{8}-7043 x^{7}+9479 x^{6}$ & \\
& $-8254 x^{5}+4533 x^{4}-1505 x^{3}+283 x^{2}-27 x+1$ & 0.0000123957 \\
68 & $x^{12}-22 x^{11}+206 x^{10}-1079 x^{9}+3493 x^{8}-7288 x^{7}+9922 x^{6}$ & \\
& $-8745 x^{5}+4867 x^{4}-1638 x^{3}+310 x^{2}-29 x+1$ & 0.0000094705 \\
69 & $x^{12}-22 x^{11}+207 x^{10}-1093 x^{9}+3573 x^{8}-7530 x^{7}+10343 x^{6}$ & \\
& $-9172 x^{5}+5109 x^{4}-1706 x^{3}+317 x^{2}-29 x+1$ & 0.0000079329 \\
& $x^{12}-22 x^{11}+205 x^{10}-1064 x^{9}+3401 x^{8}-6988 x^{7}+9354 x^{6}$ & \\
\hline 70 & $-8104 x^{5}+4441 x^{4}-1480 x^{3}+281 x^{2}-27 x+1$ & 0.0000054356 \\
\hline & $x^{13}-23 x^{12}+230 x^{11}-1313 x^{10}+4730 x^{9}-11240 x^{8}+17929 x^{7}$ & \\
& $-19217 x^{6}+13689 x^{5}-6338 x^{4}+1837 x^{3}-313 x^{2}+28 x-1$ & 0.0025727620
\end{tabular}

Note. See the online version of this paper for a link to: Some minimal trace totally positive algebraic integers (degrees 12, 13, 14)

\section{ACKNOWLEDGMENT}

The author is grateful to the referee for a careful reading of this paper, and gives thanks to both the referee and the associate editor for helpful comments.

\section{REFERENCES}

1. J. Aguirre, M. Bilbao, J.C. Peral, The trace of totally positive algebraic integers, Math. Comp. 75 (2006), no. 253, 385-393. MR2176405 (2006h:11123)

2. J. Aguirre, J.C. Peral,The trace problem for totally positive algebraic integers, Number Theory and Polynomials, London Mathematical Society Lecture Note Series 352, 1-19, Cambridge University Press, Cambridge 2008. MR2428512 (2009k:11172)

3. H. Cohen, A course in computational algebraic number theory, Springer Graduate Texts in Mathematics 138, Springer-Verlag, Berlin 1993. MR1228206 (94i:11105)

4. V. Flammang, Trace of totally positive algebraic integers and integer transfinite diameter, Math. Comp. 78 (2009), no. 266, 1199-1125. MR2476574 (2009m:11173)

5. V. Flammang, M. Grandcolas, G. Rhin, Small Salem numbers, Number theory in progress, Vol. 1 (Zakopane-Kościelisko, 1997), 165-168, de Gruyter, Berlin, 1999. MR.1689505 (2000e:11132)

6. V. Flammang, G. Rhin, Algebraic integers whose conjugates all lie in an ellipse, Math. Comp. 74 (2005), no. 252, 2007-2015. MR 2164108 (2006c:11121)

7. J. McKee, C.J. Smyth, Salem numbers of trace -2 and traces of totally positive algebraic integers, Algorithmic number theory, Lecture Notes in Computer Science 3076, 327-337, Springer-Verlag, Berlin, 2004. MR2137365 (2006a:11134)

8. MR 2105815 (2005g:11203)

9. The PARI Group, PARI version 2.3.4, Bordeaux 2006, available from http://pari.math.u-bordeaux.fr/. 
10. R.M. Robinson, Algebraic equations with span less than 4, Math. Comp. 18 (1964), no. 88, 547-559. MR0169374 (29:6624)

11. I. Schur, Über die Verteilung der Wurzeln bei gewissen algebraischen Gleichungen mit ganzzahligen Koeffizienten, Math. Z. 1 (1918), no. 4, 377-402. MR.1544303

12. C.L. Siegel, The trace of totally positive and real algebraic integers, Ann. of Math. (2) 46 (1945), 302-312. MR0012092 (6:257a)

13. C.J. Smyth, Totally positive algebraic integers of small trace, Ann. Inst. Fourier (Grenoble) 34 (1984), no. 3, 1-28. MR762691 (86f:11091)

14. 663-681. MR736460 (86e:11115)

15. _ An inequality for polynomials, Number theory (Ottawa, ON, 1996), CRM Proc. Lecture Notes 19, 315-321, Amer. Math. Soc., Providence, RI, 1999.

16. Q. Wu, On the linear independence measure of logarithms of rational numbers, Math. Comp. 72 (2002), no. 242, 901-911. MR.1954974 (2003m:11111)

Department of Mathematics, Royal Holloway, University of London, Egham Hill, Egham, Surrey, TW20 0EX, England, United Kingdom

E-mail address: james.mckee@rhul.ac.uk 